Ciencia y Educación, Vol. 4, No. 3, septiembre-diciembre, 2020

ISSN (impreso): 2613-8794•ISSN (en línea): 2613-8808

DOI: https://doi.org/10.22206/cyed.2020.v4i3.pp79-93

\title{
¿Por qué se incorpora el doctorado profesional al Programa Nacional de Posgrados de Calidad en México?
}

\author{
Why is the professional doctorate incorporated into the \\ National Quality Postgraduate Program in Mexico?
}

Josefina Patińo Salceda ${ }^{a}$ RCID: 0000-0003-2803-378X

Recibido: 24/02/2020 • Aprobado: 1/04/2020

Cómo citar: Patińo Salceda, J. (2020). ¿Por qué se incorpora el doctorado profesional al Programa Nacional de Posgrados de Calidad en México? Ciencia y Educación, 4(3), 79-93. Doi: https://doi.org/10.22206/cyed.2020.v4i3.pp79-93

\section{Resumen}

El objetivo en este artículo es responder dos preguntas sobre la incorporación del doctorado profesional al Programa Nacional de Posgrados de Calidad (PNPC), en México, en 2014: ¿cuáles fueron los factores que propiciaron la incorporación? y ¿̇cuál fue el objetivo de esta decisión? Se trabajó con un enfoque cualitativo a través del análisis de siete entrevistas semiestructuradas realizadas a expertos del tema en el país. Con los resultados se muestra que la incorporación del doctorado profesional se debe a la insatisfacción que provocaron los resultados de impacto de los doctorados de investigación en el sector productivo. Se concluye que las autoridades del PNPC intentan construir un modelo de formación diversificada con doctorados que no solo atiendan los requerimientos de la academia a través de la formación de cientificos profesionales, sino también de cientificos industriales cuyo destino laboral deseable es el sector gubernamental o empresarial. En Latinoamérica, se ha discutido poco sobre los doctorados profesionales, por lo que la experiencia de México es de gran utilidad para la discusión regional.

Palabras clave: científico; grado de doctor; industria; evaluación de la educación; profesión.

\begin{abstract}
The objective of this article is to answer two questions about the incorporation of the professional doctorate to the National Quality Postgraduate Program (PNPC) in Mexico in 2014: what were the factors that led to the incorporation, and what was the purpose of this decision? The research was carried out with a qualitative approach through the analysis of seven semi-structured interviews conducted with national experts on the subject in the country. The results show that the incorporation of the professional doctorate is due to the dissatisfaction caused by the impact results of research doctorates in the productive sector. It is concluded that the PNPC authorities try to build a diversified training model with doctorates that not only meet the requirements of the academy through the training of professional scientists, but also industrial scientists whose desirable employment destination is the government or business sector. In Latin America, there has been little discussion about professional doctorates, therefore, the experience of Mexico is very useful for regional discussion.
\end{abstract}

Keywords: scientist; doctoral degree; industry; educational evaluation; profession.

a Universidad Pedagógica Nacional, Unidad 094 Centro de la Ciudad de México. México. Correo-e: jsalceda@upn.mx 


\section{Introducción}

En México, a partir de 1991, el Consejo Nacional de Ciencia y Tecnología (Conacyt) inició la operación de un programa de evaluación y acreditación de posgrados que a través del tiempo ha sufrido varias modificaciones; la última versión es el Programa Nacional de Posgrados de Calidad (PNPC) que comenzó a operar en 2007.

De acuerdo con el último de los marcos de referencia que han orientado al PNPC, versión 6, los Doctorados Profesionales (DP) son programas asociados al desarrollo de ciertas profesiones en disciplinas o campos de conocimiento como la educación, el derecho, la administración, las ciencias de la salud, la medicina clínica, la psicología clínica y la psicología educativa, entre otras, o pueden asociarse también al desarrollo de nuevas disciplinas o interdisciplinas. Estos programas están estructurados de manera que se combinan cursos y proyectos de investigación orientada, cuyos contenidos son de naturaleza aplicada; esto con el fin de lograr una mayor relación con el lugar de trabajo. En los proyectos de tesis pueden participar como asesores los representantes de la empresa a la cual se vincula el programa de doctorado o proyecto de investigación. La dedicación de los estudiantes puede ser de tiempo completo o parcial; la mayoría son presenciales y pueden contemplar periodos para realizar prácticas profesionales. En el PNPC se reconoce que las características de estos programas son muy variadas: algunos, por ejemplo, pueden enfocarse en la formación de profesiones específicas, mientras que otros procuran una formación más general (Conacyt y Subsecretaría de Educación Superior de la Secretaría de Educación Pública (SEP), 2015).

En este artículo se analiza la incorporación de los DP en lugar de su origen, dado que estos no se originaron en México y no fue este el propósito del estudio. La experiencia internacional indica que Estados Unidos de América registró una orientación profesional en los doctorados desde 1920; no obstante, su expansión se considera relativamente novedosa, dado que la proliferación de este tipo de programas se circunscribe a la última década del siglo xx. Los datos revisados hasta el momento indican que los siguientes países que registraron DP fueron Inglaterra y Australia, pues sus primeros programas se ubican en 1991 y 1992, respectivamente (Bourner, Bowden y Laing, 2001). Por lo anterior, la incorporación de los DP al PNPC de México podría interpretarse como un acontecimiento tardío. Asimismo, es necesario resaltar que tanto en México como en otros países la incorporación de estos doctorados ha resultado polémica, porque se ha llegado a pensar que los DP son de menor calidad que los tradicionales Doctorados de Investigación (DI).

En México la categoría DP se mencionó por primera vez en el PNPC en 2014, pero esta no resultó del todo novedosa, dado que el Consejo Mexicano de Estudios de Posgrado, A. C. (Comepo), tiempo atrás había utilizado la categoría doctorado profesionalizante. Se desconoce la fecha exacta a partir de la cual se utilizó por primera vez esta categoría en Comepo, pero más allá de eso, es importante mencionar, que a diferencia del PNPC, el Comepo no cuenta con criterios de clasificación expresamente definidos para cada orientación de los programas, por lo que es imposible afirmar que el DP del PNPC es equiparable al doctorado profesionalizante del Comepo. Una segunda hipótesis es que esta última categoría puede incluir una variedad de orientaciones, pero eso deberá discutirse en otro texto.

El hecho que se debe destacar es que tanto el PNPC como el Comepo son dos instancias nacionales que cuentan con registros de doctorados distintos a los tradicionales, es decir, a los DI. En agosto de 2017 el Padrón del PNPC tenía registrados ocho DP (Conacyt, 2017), mientras que en la base de Comepo se registraron 89 doctorados profesionalizantes (Comepo, 2017a). Aunado a esto, se encuentra la hipótesis de que en el PNPC existen más DP que tuvieron que registrarse como DI porque era la única opción posible hasta antes de 2014. Estos datos indican que la existencia de doctorados distintos a los DI representa un fenómeno relativamente reciente en el país y, por lo tanto, es necesario estudiarlo. 
Por lo anterior, en este artículo se procuró responder dos preguntas: ¿cuáles fueron los factores que propiciaron la incorporación del DP al PNPC?, y ¿cuál fue el objetivo de esta decisión? Estas preguntas propiciaron la revisión de marcos teóricos sobre nuevas formas de producción de conocimientos, la vinculación universidad-empresa y el establecimiento de nuevos vínculos entre un nivel educativo y una profesión. Bajo estos referentes se interpretaron siete entrevistas realizadas a connotados expertos en el tema, quienes desde su perspectiva explicaron el significado del DP, así como los factores y objetivos implicados en su incorporación al PNPC.

El artículo se divide en cinco apartados: en el primero se presentó la introducción; en el segundo, el marco de referencia en el que se revisan los factores que influyen en la educación doctoral; en el tercero se describen los pasos metodológicos que guiaron el estudio; en el cuarto se presentan los resultados que responden al por qué y para qué se incorporan los DP al PNPC de acuerdo con la opinión de los entrevistados y, en el quinto apartado, se presentan la discusión y conclusiones del artículo.

\section{Marco de referencia}

\subsection{Nuevas formas de producción de conocimientos}

La idea de universidad se está definiendo actualmente bajo la influencia de procesos macro, entre los que destacan la globalización, el nuevo capitalismo y los nuevos modos de producción de conocimiento. Estos cambios también impactan en la educación doctoral y en la profesión asociada a esta. Para iniciar es necesario revisar términos como el de globalización, entendido como una nueva forma de capitalismo que impacta a la economía mundial. Esta se entiende como un conjunto de elementos que representan el crecimiento moderno mediante: "inventos, innovaciones, nuevos productos, patentes, diseños, ingeniería, mejoras del proceso productivo y administrativo, información, conocimientos, ideas, salud, entrenamiento, aprendizaje, experiencia, educación, investigación y desarrollo (ID)" (Rodríguez, 2009, p. 24).
Así, el conocimiento y la innovación adquieren gran importancia para los gobiernos nacionales y otras organizaciones que buscan institucionalizar su desarrollo a través de sistemas nacionales de innovación, entendidos como "una red de instituciones públicas y privadas, donde tiene lugar la producción, distribución y uso de nuevo conocimiento y tecnología" (Eriksson y Lindfords, citado en Almanza y Hernández, 2009, p. 155). La concepción de estos sistemas transforma la idea tradicional de universidad, de manera que esta pasa de ser productora y difusora del conocimiento, a una nueva organización que forma parte de los sistemas nacionales de innovación, y que es capaz de generar ventajas competitivas y transferir conocimiento entre los diferentes actores de la sociedad, buscando que el sector productivo obtenga beneficios derivados de estos procesos (Almanza y Hernández, 2009, p. 157).

Estos sistemas están asociados también a nuevas tendencias en la producción de conocimientos que, en su conjunto, se denominan Modo 2, en oposición al Modo 1, que se utiliza para hacer referencia a la forma tradicional de producción de conocimiento. El Modo 2 se refiere a un nuevo tipo de conocimiento y a una nueva forma de producirlo, distinguiendo su contexto, su organización, el sistema de recompensas que se utiliza y el control de calidad de la investigación (Gibbons et al., 1997). Aunque los especialistas que describen estas tendencias se abstienen de emitir juicios sobre el mejor modo de producción de conocimientos, en los espacios académicos y de política educativa, las características del Modo 2 son valoradas como un modelo a seguir. En síntesis, el Modo 2 implica que:

a) Los problemas de investigación no se definen en la academia, sino que están determinados por la utilidad que puedan generar para la industria, el gobierno o algún otro sector de la sociedad. Por ello se opta por la investigación aplicada.

b) Los actores que participan en la producción de conocimiento son más heterogéneos; no solo participan científicos. 
c) El contexto de producción de conocimiento es el mismo en el cual se pretende aplicar, es decir, en ambientes donde opera la oferta y la demanda; esto significa que puede o no producirse en las universidades.

d) La calidad de los conocimientos producidos se valora favorablemente si estos contribuyen a la solución de un problema, si el conocimiento es competitivo en el mercado, si es efectivo en cuanto a costos y si es socialmente aceptado. Esto implica que en la valoración de la calidad intervienen expertos con intereses intelectuales, pero también económicos, políticos y sociales (Gibbons et al., 1997).

Con relación a las universidades, algunos de los valores asociados a la nueva idea de universidad han sido cuestionados por De Sousa (2004), Fernández (2009) y Stromquist (2009), quienes identifican estos cambios con el denominado capitalismo académico, el cual, desde su punto de vista, vulnera la autonomía universitaria. Más allá de esa discusión, se reconoce que estos cambios en la idea de universidad y en las formas de producción de conocimiento también han tenido impacto en la profesión académica (Stromquist, 2009; Teichler, 2015).

\section{2. ¿Por qué se incrementan los DP en otros paises?}

De acuerdo con la revisión de la literatura, en países de Latinoamérica, con excepción de Brasil, sigue existiendo una fuerte orientación académica del doctorado, por lo que se continúa considerando un solo tipo de doctorado con un perfil de egreso orientado al binomio investigación-docencia y, por ende, a la carrera académica (Núnez-Valdés, González y Sarzoza, 2019; Núñez-Valdés y González, 2019; Patiño, 2019). Mientras que, en países europeos y asiáticos se identifican nueve tipos: “a) de investigación, b) profesional, c) de enseñanza, d) el $\mathrm{PhD}$ por trabajo publicado, e) doctorado basado en la práctica, f) el doctorado de nueva ruta, g) interinstitucional, h) de cooperación, e i) industrial" (Bao, et al., citado en Patiño, 2019).
Cada vez es mayor el número de estudios que se refieren al incremento de los DP, sobre todo en Estados Unidos de América, Inglaterra, Australia, Canadá, China e Islandia (Bourner et al., 2001; Chiteng y Hendel, 2012; Cuthbert y Molla, 2015; Lester, 2007; Loxley y Seery, 2012; Taylor, 2004; Wildy et al., 2015). En estos estudios se señala que el $\mathrm{DP}$ se creó como una alternativa al $\mathrm{PhD}$. Aunque se sabe que existe una gran variedad de enfoques sobre los estudios de doctorado (Kehm y Teichler, 2016; Patiño, 2019), y que estos varían de acuerdo con el país y la disciplina (Teichler, 2015) pero, las razones por las que se incrementan los DP son similares a las que se identifican en otros países.

Por ejemplo, en el estado del arte que realizan Wildy, Peden y Chan (2015) identifican once razones asociadas al incremento de los DP: a) el deseo de las universidades por volverse cada vez más social y económicamente relevantes; b) la crítica hacia los doctorados tradicionales por apartarse del mundo del trabajo y generar una limitada gama de herramientas en los graduados; c) una exigencia de las políticas gubernamentales por incrementar la educación de la sociedad y ser altamente competitivos económicamente; d) el alto porcentaje de deserción de los programas de doctorado tradicionales y la necesidad de programas más estructurados, como los DP; e) la presión que enfrentan las IES para proveer programas que reúnan las necesidades de un doctorado con creatividad artística; f) los DP se basan más en investigación aplicada que en investigación pura; g) algunas universidades ofrecen DP porque les permite tener más estudiantes; h) la masificación de la educación superior; i) el desarrollo profesional y el prestigio de las profesiones; mayor legitimidad al aprendizaje obtenido en el lugar de trabajo; k) el $\mathrm{PhD}$ se considera insuficiente para el conocimiento requerido en los lugares de trabajo modernos.

Las razones identificadas por Wildy et al., (2015) se sintetizan, a su vez, en cuatro aspectos: la crítica hacia el formato de los doctorados tradicionales; las exigencias de la política gubernamental asociadas a la competitividad y la economía; la búsqueda 
de prestigio por parte de algunas profesiones y la legitimidad del aprendizaje dual (escuela y trabajo).

Más allá de intentar demostrar cada una de las razones que identifican los autores se reconoce que el doctorado, como nivel educativo, enfrenta fuertes críticas respecto a su pertinencia, fundadas principalmente en el destino laboral de los graduados de doctorado.

\subsection{Dos tipos de cientificos}

Tradicionalmente, se había considerado que el doctorado era el nivel educativo que formaba científicos; con algunas excepciones, referirse a ellos era casi igual que referirse a los académicos. Sin embargo, las transformaciones en la idea de universidad y en los sistemas de producción de conocimiento crearon la necesidad de distinguir dos tipos: el cientifico profesional, que busca "promover la investigación básica libre de todo compromiso de índole comercial”, y el cientifico industrial, que “(...) difiere radicalmente del profesional en la medida en que su interés primordial radica en la eficiencia práctica; en torno a ella se formulan problemas, se revisan teorías, se innovan métodos y se aplican las técnicas más adecuadas" (Kornhauser citado en Pacheco, 1997, pp. 153-154) [las cursivas son del autor]. De esta forma se asociaría al científico profesional con el Modo 1 de producción de conocimiento; y al científico industrial con el Modo 2.

Bourner et al. (2001) realizaron una amplia descripción de la formación en un $\mathrm{PhD}$ — también denominado doctorado tradicional- y un doctorado profesional. Mientras que al primero lo vinculan a los investigadores profesionales (professional researchers), al segundo lo asocian con los profesionales que investigan (researching professionals), de manera que por primera vez se contempla una profesión distinta vinculada a los doctorados. Los autores señalan un conjunto de características que distinguen a ambos tipos de formación, pero son dos las que podrían sintetizar la diferencia. El doctorando de un $\mathrm{PhD}$, por ejemplo, tiene interés en hacer carrera académica y en realizar investigación básica, principalmente; en cambio, el doctorando de un programa profesional tiene interés en realizar investigación útil para mejorar su práctica profesional en la vida laboral y, por lo tanto, se orienta al desarrollo de la investigación aplicada. Esta distinción concuerda con la referida por Kornhauser (citado en Pacheco, 1997). A pesar de que existe cierta claridad en la distinción anterior, esta no se ha generalizado, pues existe cierta resistencia para reconocer que los DP son tan valiosos como el PhD (Schildkraut y Stafford, 2015).

Para el caso de México, Pacheco (1997) sostiene que la base institucional de la investigación universitaria permitió la coexistencia de estos dos ordenamientos de la actividad científica, sin embargo, reconoce que el científico industrial no se definió en la política institucional como sí se hizo con el científico profesional, el cual se ha vinculado tradicionalmente al doctorado orientado hacia la investigación.

En términos de Sarfatti (1988) el incremento de los DP estaría asociado a un movimiento de reforma profesional que busca vincular una nueva profesión a un nivel educativo y un trabajo, es decir, al científico industrial conectado al doctorado, esperando que este se vincule a un nuevo mercado de trabajo. No obstante, se sabe que un movimiento de reforma profesional o cambio planificado (Gómez y Tenti, 1989) no están exentos de conflictos, dado que en la historia de estos movimientos se identifica “(...) la existencia de comunidades de profesionales que actúan de forma excluyente, es decir, asegurando el monopolio del conocimiento y de la práctica profesional a aquellos que han sido acreditados debidamente" (Finkel, 2015, p. 160).

\subsection{El contexto mexicano de los estudios doctorales}

En México, el doctorado carece de estudios específicos que permitan distinguir qué tipo de investigación ha estado presente en la formación. No obstante, existen otras investigaciones y datos del Conacyt que muestran que el doctorado se ha orientado de manera predominante a la formación del científico profesional y, por ende, a la investigación básica por encima de la aplicada. 
En 2010, una investigación sobre la vinculación entre universidad-empresa, basada en la Encuesta Nacional de Vinculación a Instituciones de Educación Superior (Enavi) y la Encuesta Nacional de Vinculación a Empresas, se reconoce como "(...) una debilidad estructural la pobre intensidad de vinculación entre empresas e instituciones educativas y de investigación" (Cabrero, 2012, p. 9). Uno de los hallazgos de esta investigación muestra que:

Del grupo de empresas entrevistadas que realizan actividades de investigación y desarrollo, apenas 26 por ciento realiza actividades de investigación y desarrollo en colaboración con IES; 12 por ciento reporta colaboración con instituciones de educación media superior técnica; y 8 por ciento reporta colaboración con instituciones de formación para el trabajo. La mayor parte de empresas de este grupo, alrededor de 54 por ciento, realiza su colaboración con despachos de consultoría, fundaciones científicas, organismos internacionales, profesionales, $\mathrm{u}$ otras organizaciones privadas (Arellano, 2012, p. 255).

Lo anterior significa que el sector productivo no está aprovechando la capacidad instalada en las IES para realizar proyectos de investigación con beneficios comunes. Esto quiere decir que el Modo 2 es una tendencia incipiente en el país. Aunado a lo anterior, se ha identificado que en varios países el principal destino laboral de los graduados de doctorado son las propias IES con datos que van del 40 al 80 por ciento (Auriol, 2010). En el caso de México, no se cuenta con datos confiables al respecto, pero se sabe que muchos profesores universitarios obtuvieron su grado de doctorado gracias a las becas otorgadas por del Programa de Mejoramiento del Profesorado (Promep), ahora Programa para el Desarrollo Profesional Docente para el Tipo Superior (Prodep), esto significa que el doctorado ha tenido como función principal la formación de los docentes universitarios.

Es importante mencionar que la labor del cientifico profesional en México ha estado regulada por el Sistema Nacional de Investigadores (SNI), programa de estímulos a cargo del Conacyt. Aunque este programa es polémico por los criterios usados para decidir quién debe ser admitido como miembro, goza de mucha aceptación y ha operado por más de 30 años, dado que se creó en 1984. También es cierto que el SNI se ha incrementado en gran medida por los graduados de doctorado de programas con registro en el PNPC.

El marco de referencia presentado aquí permite identificar que las tendencias en las nuevas formas de producción de conocimientos no han impactado todavía a la educación doctoral, ya que los últimos datos indican que el doctorado en el país se ha caracterizado por su orientación académica, pues este nivel educativo se ha vinculado al cientifico profesional, en el mejor de los casos, más que al científico industrial habilitado para insertarse en ámbitos productivos.

\section{Metodología}

Los resultados que se presentan en este artículo forman parte de un proyecto mayor sobre el significado, función y procesos de formación de los doctorados profesionales. Específicamente, este artículo presenta un estudio exploratorio que buscó analizar por qué y para qué se incorporan los Doctorados Profesionales (DP) al Programa Nacional de Posgrados de Calidad (PNPC), lo cual se sustenta en la aplicación de siete entrevistas semi estructuradas a expertos del tema en el país. Vale la pena recordar que el PNPC es el programa mediante el cual se evalúan y acreditan los posgrados en México y goza de gran aceptación entre la comunidad académica.

\subsection{Selección de expertos}

Primero se seleccionó a dos expertos: uno de ellos tiene influencia en la toma de decisiones sobre el PNPC y otro se desempeńó como miembro del Comité Directivo del Comepo. Posteriormente, se utilizó la técnica de bola de nieve, es decir, se pidió a los informantes que recomendaran a otros expertos en el tema, con la única condición de que tuvieran visiones 
diversas sobre el doctorado. Así se logró la participación de expertos que se han desempeñado como académicos, administrativos (o ambos) y consultores. Los informantes fueron seis hombres y una mujer; en el código de clasificación se omite la referencia al sexo con el propósito de resguardar el anonimato de la única mujer. Actualmente, los informantes laboran en la Universidad Nacional Autónoma de México (UNAM), en el Conacyt, el Comepo, el Instituto Potosino de Investigación Científica y Tecnológica (IPICyT), la Universidad Iberoamericana y en Esmart Consultores, S. C.

Como suele ocurrir en los estudios cualitativos, el númeroy tipo de informantes no se eligieron buscando representatividad; en su lugar, se seleccionaron informantes que contaran con conocimientos sobre el tema y que estuvieran suficientemente involucrados en la toma de decisiones en el PNPC, o que, en su defecto, estuvieran lo bastante cerca de este proceso como para compartir sus opiniones respecto al tema de esta investigación.

\subsection{Guion de entrevista}

Para realizar las entrevistas se diseñó un guion general que contempló la exploración de ocho categorías sobre los doctorados profesionales: a) significado y relevancia; b) origen y fundamentos de la orientación profesional; c) cómo y quiénes decidieron crear la nueva orientación; d) criterios de evaluación; e) alcances del PNPC; f) prioridades nacionales respecto al área de conocimiento y; g) pertinencia.

Las entrevistas duraron entre una y dos horas. Posteriormente se transcribieron y se enviaron a los expertos para validar su contenido, de manera que algunos de los participantes hicieron precisiones a las transcripciones.

\subsection{Análisis y exposición de los resultados}

Los textos se analizaron con el software Atlas ti. Cada transcripción fue clasificada asignando un código compuesto por la letra E (inicial de entrevista) y el número de entrevista, de acuerdo con el orden en que se realizaron.

Con la finalidad de facilitar la exposición y comprensión de los resultados de investigación se realizó una nueva propuesta de categorías que sintetiza las que se utilizaron en el guion de entrevista.

\section{Resultados}

En este apartado se presentan los resultados del estudio, derivados de las opiniones de los expertos acerca de las siguientes preguntas: ¿por qué se incorporan los doctorados profesionales en el PNPC?, y ¿qué se espera lograr a través de esta decisión? Al realizar este estudio se pudo ver que los entrevistados tienen una idea positiva de los DP. Se reconoce, sin embargo, que otros informantes podrían brindar una mirada distinta respecto a las preguntas planteadas. El valor de esta investigación radica en la exposición y análisis de las explicaciones que los informantes, en calidad de expertos - y en algunos casos, de tomadores de decisión-, brindaron respecto a la incorporación de los DP.

\section{1 ¿Por qué se incorporan los DP al PNPC?}

Uno de los entrevistados señala que la incorporación de los DP surgió por iniciativa de la propia Dirección de Posgrados del Conacyt, después de poner en marcha la modalidad de posgrados con la industria (E1). ${ }^{a}$ Sin embargo, otro de los expertos considera que el Comité Directivo de Comepo, en representación de sus miembros, tuvo cierta influencia en dicha decisión, pues en distintas ocasiones hubo disposición por parte del director de Posgrado del Conacyt para discutir los criterios de

a. Esta modalidad se creó en la Dirección de Posgrados, y se puso en marcha en 2012, es decir, dos años antes que los DP. 
evaluación. Por otra parte, existe la percepción de que el director de Posgrado tomó en cuenta algunas sugerencias del Comité Directivo de Comepo. Uno de los entrevistados señala que la incorporación de los DP forma parte de una serie de reformas iniciadas a partir del 2012, fecha en la que se crearon marcos de referencia específicos para evaluar a los posgrados con la industria, las especialidades médicas $y$, posteriormente, los posgrados en modalidad no escolarizada (E2).

Algunos expertos consideran que el doctorado de investigación ha tenido una fuerte orientación académica que se manifiesta en un ideal sobre el perfil del egresado. Este ideal se construyó bajo las estructuras académicas de las universidades, principalmente las públicas, en las cuales existen criterios de organización asociados a las áreas de conocimiento, disciplinas, facultades o departamentos. De esta manera, se generalizó la idea de que en el doctorando se prepara para convertirse en científico académico o científico profesional. Los entrevistados consideran que el tipo ideal es el propio director de tesis, es decir, un profesorinvestigador que se dedica principalmente a realizar investigación básica (en la mayoría de los casos), y cuya productividad se refleja en publicaciones, preferentemente arbitradas, así como en la docencia universitaria y otras actividades académicas. De acuerdo con algunos expertos, la principal función de los doctorados de investigación ha sido la "clonación de investigadores" (E6). Con ello se refieren a que la formación del doctorando está basada en la reproducción de las prácticas y valores de los propios directores de tesis.

Por otra parte, otros expertos opinan que el conflicto -o el descontento- con los DI surge en la medida en que se reconoce que la sociedad, y el Sistema Nacional de Ciencia, Tecnología e Innovación (SNCTI), demandan actualmente nuevas formas de producción de conocimientos. Por esta razón, algunos expertos identifican cambios en la percepción sobre el tipo de investigación más pertinente para la sociedad.

Al mismo tiempo, otro entrevistado afirma que la imagen del científico profesional ha estado alejada de la investigación aplicada porque existe una percepción negativa sobre esta. Un entrevistado señala que el problema consiste en "pensar que el conocimiento aplicado es menos importante que la generación de conocimiento, [esto] es un problema de apreciación realmente importante que se basa en prejuicios ideológicos" (E2). Además de la percepción hasta cierto punto negativa de la investigación aplicada, está el rechazo a los investigadores que la realizan. De acuerdo con uno de los especialistas:

Si tú hablas de esto con el académico que tiene más de 20 o 30 años en la academia, te comentaría que ha habido épocas en las que se pensaba que una persona que resolvía problemas, que hacía proyectos para la industria, que recibía dinero siendo académico, que recibía dinero por un problema de la industria, era visto como si se estuviera prostituyendo, era una cuestión así, de pureza. [Pensaban]: "nosotros somos puros y nada más vamos a resolver problemas de la academia” (E3).

Uno de los expertos señala que el problema se evidencia en el marcado desequilibrio que existe en la productividad derivada de los programas de doctorado de investigación: en donde “(...) alrededor del $96 \%$ de los productos de investigación derivan de la generación de conocimiento, y solamente un $4 \%$ de la totalidad de los programas se traduce en aplicaciones" (E1). Otro entrevistado señala: “[...] se parte del hecho de que una maestría y un doctorado están diseñados para solucionar problemas locales, estatales, nacionales o internacionales; pero esta asociación no se observa" (E5).

Algunos entrevistados coinciden en señalar que la incorporación de los DP se asocia al reconocimiento del valor de la investigación aplicada y a la creación de las estructuras institucionales que la legitiman. Por lo menos así lo sugiere el comentario de uno de los entrevistados: "Yo creo que el hecho de que se cree una ventanilla más para los doctorados profesionales es sencillamente para reconocer que 
estamos en una etapa donde el conocimiento que se aplica, el de más alto nivel, tiene mucha relevancia para la sociedad" (E3).

Los expertos opinan que la sociedad actual demanda que las investigaciones que se realicen tengan un impacto concreto en algún sector de la sociedad. Consideran que el doctorado de investigación ha contribuido con la reproducción y consolidación de plantas académicas y comunidades científicas, pero ha sido ajeno a los problemas concretos que enfrenta la sociedad. En síntesis, existe consenso entre los expertos en señalar que la incorporación de los DP se debió al reconocimiento explícito de la necesidad de contar con otro tipo de doctorado que cubriera las funciones que los DI no cubrían. Estas funciones son el desarrollo de la investigación aplicada y la innovación.

Además del reconocimiento de la importancia de la investigación aplicada, los expertos señalaron que la incorporación de los DP también está asociada a la necesidad de vincular a este tipo de formación con otros mercados laborales aparte del académico. Algunos de los expertos entrevistados aseguran que, si bien no existen cifras precisas, haciendo algunos cálculos sobre el número de graduados de doctorado y el número de plazas disponibles en la educación superior del país queda claro que los espacios laborales en la academia son insuficientes para recibir a todos los graduados de doctorado. Así lo explica uno de ellos:

Una persona que estudia doctorado, prácticamente tiene su nicho de trabajo dentro de una universidad o dentro de una institución de educación superior que le permite hacer docencia e investigación. Entonces hay una situación desde hace más de cinco o diez años, en la cual se nota una saturación de plazas, de manera no uniforme, me refiero a las principales instituciones (...) las que serían la primera opción de empleo de muchos egresados de doctorado que desean iniciar su vida profesional (E3).

Otro de los entrevistados hace los siguientes cálculos:
(...) la SEP es la dependencia que proporciona los recursos al sector académico más importante de este país, es decir, está creando, desde hace tres años, tres mil plazas para los nuevos egresados de doctorado; Conacyt crea alrededor de unas 500 plazas, entre las cátedras y las plazas para los centros Conacyt, que son 27; y el sector privado, nosotros suponemos, porque no hay información respecto a eso, está creando alrededor de 500 y 700 plazas (E1).

El argumento sobre la insuficiencia de plazas en educación superior para los graduados de doctorado se complementa con la idea de que los graduados de los DP estarían mejor preparados para insertarse en mercados laborales del sector industrial y gubernamental.

Además de la vinculación del doctorado a otros mercados laborales también se encuentra presente el creciente interés de profesionales de disciplinas como administración, contaduría, psicología y educación de realizar un posgrado (E1). Esta afirmación se complementa con algunos datos que evidencian un gran incremento de graduados de doctorados en educación y en otras disciplinas del área de ciencias sociales, administración y derecho.

\section{2 ¿Qué se espera lograr con los DP?}

Los expertos advierten que la incorporación de los DP al PNPC tiene al menos dos objetivos: el primero se relaciona con un nuevo modelo de doctorado, y el segundo con la legitimación de este. El nuevo modelo está basado en la pertinencia de los estudios de doctorado a través de la diversificación de orientaciones y modalidades para satisfacer las demandas de todos los sectores de la sociedad. En la opinión de los entrevistados, el doctorado de investigación resolvía solo una parte de las necesidades de la sociedad a través de la formación de cientificos profesionales; por lo tanto, era necesario crear otras opciones de formación que atendieran la demanda del sector empresarial, gubernamental y social. El segundo objetivo se relaciona con la necesidad de legitimar algunas prácticas que ya 
existían en las comunidades académicas, pero que tenían dificultades para lograr su aceptación.

Se abordan primero las opiniones de los expertos respecto al modelo de formación. Algunos expertos mencionaron que el DP es parte de un modelo de formación centrado en la pertinencia, porque se espera que atiendan las necesidades del sector productivo, es decir, aquellas que usualmente los DI no han podido resolver:

(...) un doctorado profesional es el que está formando a una gente con ese nivel, lo capacitan para negociar, su enfoque se orienta a la solución de problemas y se va a incorporar al sector productivo, predominantemente. El graduado de este doctorado podrá ser docente y desempeñar otras actividades académicas, pero principalmente se va a insertar en el sector productivo (...) (E3).

Es importante mencionar que algunos expertos identifican similitudes entre el DP y el doctorado con la industria (esta modalidad se dirige a profesionales en activo que requieran formarse en ingeniería y tecnología (Conacyt y Subsecretaría de Educación Superior de la SEP, 2012), ya que ambos fueron creados, principalmente, para vincular a los graduados con el sector empresarial. Es con base en esta semejanza que las autoridades del PNPC proponen un modelo de formación doctoral basado en la oferta de programas con dos orientaciones (investigación y profesional) y tres modalidades (posgrados con la industria, escolarizada y no escolarizada).

El segundo objetivo de la incorporación de los DP fue la legitimación de ciertas prácticas. Los expertos coinciden en señalar que dichos doctorados existían antes de que se creara la categoría de DP en el PNPC; es decir, que ya existían programas de doctorado en los que se realizaba investigación aplicada y en donde los participantes se vinculaban con la industria u otros sectores de la sociedad. En opinión de algunos expertos, el problema radicaba, por un lado, en la inexistencia de las condiciones estructurales necesarias para que la investigación aplicada y la vinculación se realizaran de manera natural. Y, por el otro, a que a las actividades que se realizaban en esta vertiente no se les reconocía el mismo prestigio que a las de los doctorados de investigación. Las condiciones a las que los expertos se refirieron se resumen en tres aspectos: a) las normatividades de las IES; b) la evaluación de la profesión del cientifico profesional y c) la asociación entre el PNPC y el Sistema Nacional de Investigadores (SNI).

Los entrevistados consideran que los programas de doctorado han estado limitados por la falta de flexibilidad de las instituciones y la carencia de normativas que contemplen la vinculación formal fuera de la academia. Así lo explica un entrevistado: "Las instituciones de educación superior, sobre todo las públicas estatales, tienen reglamentos muy rígidos y esta situación hace difícil establecer un convenio con las empresas; les genera muchos problemas" (E7). Además de las dificultades relacionadas con los aspectos formales de la vinculación entre instituciones e industrias, los entrevistados señalan la diferencia que existe entre el tiempo en la academia y el tiempo en la industria respecto de la obtención de resultados, ya que, aseguran, tienen lógicas distintas (E3).

La normatividad de las IES, reconocida por su rigidez, se combina con los valores que predominan en la evaluación de la profesión científica a través del SNI. De acuerdo con los expertos, la vinculación entre el cientifico profesional y otros sectores de la sociedad no tiene tanta importancia dentro de los criterios de evaluación del SNI como la publicación de artículos, capítulos de libro, libros u otro tipo de producción escrita y difundida en revistas o editoriales de prestigio. De acuerdo con algunos entrevistados, esta situación moldea las actuaciones de los científicos que desean ser parte del SNI, pues sus esfuerzos se centran en la producción de publicaciones, en vista de que las actividades de vinculación son problemáticas y de poco valor en el Sistema (E6-E7).

Más allá del impacto del SNI en las prácticas del cientifico profesional, algunos entrevistados señalan que este Sistema, en su función de órgano regulador de la profesión científica, controla los límites de esta al establecer las pautas sobre quién es o no digno de merecer la distinción de investigador nacional. 
Uno de los expertos explica: "A los investigadores de ciencias sociales se les aplicaban criterios que correspondían a las ciencias duras; esto se debió a que el SNI se creó por investigadores de las ciencias duras" (E4). De esta manera, se señala que el SNI ha incentivado principalmente el reconocimiento de científicos de disciplinas en las que predomina la investigación básica y ha relegado aquellas en las que se realiza investigación aplicada —o tienen interés en realizarla-, es decir, las que corresponden a campos profesionales. Así lo menciona un entrevistado:

Nosotros nos dimos cuenta de que en el SNI, por ejemplo, se encuentran miembros que son contadores y administradores que realizan un trabajo profesional pero son muy pocos porque, evidentemente, los comités de selección del SNI son totalmente académicos (E1).

Adicional a lo anterior, algunos entrevistados reconocen que la clasificación de las disciplinas es cada vez más difícil por el surgimiento de la interdisciplina y las nuevas disciplinas; es por ello que plantean la importancia de revisar las clasificaciones actuales en relación al SNI y su asociación con el PNPC.

Algunos de los entrevistados sugieren que el funcionamiento del SNI afecta el reconocimiento de ciertos doctorados en el PNPC. Como se sabe, uno de los criterios de evaluación de los programas que tiene un peso importante es el porcentaje de miembros del SNI en los núcleos académicos. Así, uno de los expertos sugiere la participación de otras organizaciones como interlocutores de los programas:

(...) y entonces nos dimos cuenta de que podíamos plantearlos, de una manera similar a la que lo hicimos con los posgrados con la industria, pero usando como interlocutores externos a los gremios; es decir, asociaciones y colegios de ingenieros, esto sería una liga perfecta. Si la UAEM quiere tener posgrado profesional, un doctorado en ingeniería, puede tener un doctorado de esa categoría si está relacionado con la academia y con el colegio de ingenieros mecánicos electricistas, de ingenieros civiles, etcétera; ellos son los interlocutores (E4).

Como se ha podido ver, los expertos entrevistados para este estudio destacan distintos aspectos involucrados a la incorporación de los DP.

\section{Conclusiones}

La intención en este artículo fue contestar a las preguntas relacionadas con el por qué y el para qué de los Doctorados Profesionales (DP) en el Programa Nacional de Posgrados de Calidad (PNPC), considerando la opinión de expertos en el tema. Sin embargo, antes de plantear las conclusiones a las que se llegó con la investigación, es necesario reconocer que los resultados representan una visión limitada sobre la cuestión, pues únicamente se entrevistaron académicos o administradores que se manifestaron a favor de la incorporación del doctorado profesional. Por otra parte, si bien el aporte de este artículo radica en presentar una visión general respecto al problema de investigación, será necesario realizar otras investigaciones que analicen su impacto por disciplina o área de conocimiento, así como por tipo de institución.

Los resultados permiten confirmar que el doctorado, con sus dos orientaciones se encuentra delimitado por las políticas de la educación superior y las de ciencia y tecnología, tal como señala la literatura, pero se debe agregar un elemento que no solo involucra a estos sistemas, sino que también involucra a la estructura de las profesiones, y a los sistemas de control de estas, en este caso, el SNI. Por lo tanto, se concluye que la incorporación de los DP forma parte de un fenómeno complejo integrado por el propio proceso de formación, el vínculo entre este nivel y las profesiones, y la forma en que estas son controladas por los propios miembros de los gremios y las agencias de política.

Los resultados sugieren que la asociación entre el PNPC y el SNI empieza a tomar distancias por la falta de convergencia entre los objetivos de cada uno. Las implicaciones concretas de estas distancias 
se presentan en los criterios para evaluar la calidad del personal académico de los programas de doctorado. Para los DI, sigue siendo necesario que el Núcleo Académico Básico cuente con cierto porcentaje de miembros con reconocimiento del SNI, en cambio, para los DP ya no es un requisito; para este último, importa la evidencia de un ejercicio profesional de relevancia, en este caso, el comité evaluador del programa es responsable de valorar este aspecto con base en los medios de verificación que se presentan en la evaluación (Conacyt y Subsecretaría de Educación Superior de la SEP, 2015).

En este sentido, el Comepo toma partido en apoyo a las propuestas del PNPC y lo hace público a través de la Segunda Declaratoria de Morelia sobre el posgrado nacional, emitida el 22 de septiembre de 2017 en el Xxxi Congreso Nacional de Posgrado. En esta se reclama al SNI el uso de criterios de evaluación que privilegian indicadores bibliométricos tradicionales, en detrimento de la pertinencia, principal objetivo del nuevo PNPC (Comepo, 2017b). Este reclamo sugiere la conveniencia de que el SNI modifique sus criterios de evaluación para incluir con mayor peso la innovación y la vinculación encaminadas a resolver problemas complejos. En el fondo de esta discusión se hallan algunas cuestiones que deberán estudiarse con mayor profundidad, por ejemplo, ¿sería conveniente distinguir entre investigadores profesionales y profesionales que investigan?, ¿ambos debieran considerarse dentro del sistema con derechos equiparables en cuanto a estatus y estímulos? Estas no son cuestiones banales, los resultados del estudio dejan ver una pugna por mayor estatus y acceso a recompensas, sin embargo, las implicaciones del cambio todavía son poco claras, pues esta situación no se ha discutido lo suficiente.

Respecto a la pregunta ¿por qué surgen los DP?, la respuesta se podría sintetizar en dos aspectos: el primero se refiere a la insatisfacción que las autoridades y otros actores tienen respecto de los resultados de los DI, pues reconocen que en estos programas no se logra formar a científicos con perfiles diversos que sean capaces de realizar tanto investigación básica como aplicada; se piensa, en resumen, que los doctorados tradicionales han tenido una orientación académica, y que sus vínculos laborales apuntan de manera predominantemente hacia la educación superior, contrario a lo que se establece en las políticas de ciencia, tecnología e innovación, cuyo interés es la formación de científicos capaces de impactar con su quehacer en el ámbito gubernamental e industrial, además del académico.

El segundo aspecto es el argumento de que las plazas de profesor-investigador ya no son suficientes para los graduados de los DI. Siguiendo el razonamiento de uno de los entrevistados, cada año se estarían generando entre 4,000 y 4,200 plazas en el sector académico. Si se asume que todos los graduados de doctorado tienen intereses laborales en este sector, entonces la oferta de plazas no sería suficiente para atender la demanda de todos los graduados, pues se sabe que en el ciclo 2014-2015 estos fueron 5,500 (Patiño, 2016). Aun cuando estas cifras deben ser consideradas con ciertas reservas, en general, el cálculo parece acertado.

Otro asunto que probablemente influyó en la incorporación de los DP, pero que no fue expresado de manera consensuada por los expertos, es la elevada matrícula que existe en educación, psicología, derecho y administración, esto significa que el número de graduados de doctorado en estos campos seguirá creciendo y, por lo tanto, crecerá también la demanda de espacios laborales con mayor estatus.

Respecto a la pregunta ¿qué se espera lograr con los DP?, la conclusión se sintetiza en dos aspectos: el primero se refiere a la creación de un nuevo modelo de formación en la educación doctoral en general, esto es, un modelo en el que se reconoce la importancia de la diversidad y de la pertinencia. Este modelo no solo incluye a los DP; la diversidad va en el sentido de ofrecer distintos enfoques para los programas: uno de ellos, el de investigación, está centrado en la investigación básica de manera predominante, cuyo objetivo es la formación de científicos profesionales; el otro está abierto a la posibilidad de que los doctorandos reciban formación dual, en la escuela y el trabajo, con énfasis en el desarrollo de investigación aplicada, 
que contribuya a resolver problemas concretos de cualquier sector de la sociedad, así el objetivo es la formación de cientificos industriales o profesionales que investigan. Además de estos dos enfoques, se encuentra la creación de nuevas modalidades, lo que permite que personas en distintas condiciones respecto a la edad, experiencia, tiempo disponible y ocupación, puedan cursar un doctorado escolarizado, no escolarizado o en combinación con la industria.

Es importante aclarar que la visión del Director de Posgrados del PNPC no concibe una orientación rígida para el doctorado, en su lugar, espera que en ambas orientaciones sea posible el desarrollo de investigación básica y aplicada, innovación, vinculación, y solución de problemas. No obstante, era importante hacer la distinción entre las orientaciones para legitimar prácticas poco comunes, pero claves en la política de ciencia, tecnología e innovación (Patiño, 2017).

Con dicho modelo no solo se diversifica la orientación y modalidad de los programas que aspiran a obtener el registro en el PNCP; sino también el perfil de los miembros de la planta académica de los programas de doctorado, pues tanto en los DP como en los doctorados con la industria podrían participar investigadores o profesionales que no pertenecen al sector académico. En el fondo, este nuevo modelo de doctorado estaría orientado a reconocer las bondades y dificultades de la formación dual.

$\mathrm{El}$ segundo aspecto que se espera obtener con los $\mathrm{DP}$ es la legitimación de prácticas y valores que no han sido suficientemente reconocidos. Tal vez este sea el aspecto más delicado de la incorporación de los DP, puesto que conduce a cuestionar ¿qué es realmente la calidad en un programa de doctorado?, ¿quién debe participar en las evaluaciones de estos programas?, y ¿cuáles serían los parámetros más adecuados para evaluarlos? Aunque estas preguntas no son novedosas, probablemente resurgirán con mayor fuerza, puesto que el asunto involucra discusiones muy antiguas respecto al tipo de investigación que se pretende validar y, ahora, respecto al tipo de científico cuyo desarrollo se procura incentivar.
Con el apoyo de la literatura analizada se hace evidente que las razones que permitieron la incorporación de los DP en otros países son cercanas a las que se presentan en este estudio. Esto permite observar que los DP no son una idea aislada, sino que surgen en un contexto en el cual la universidad, los sistemas de producción de conocimiento y la profesión científica se están transformando. Será importante que, en Argentina, Chile, Brasil, Colombia y otros países de la región consideren la discusión aquí planteada para analizar la pertinencia en la oferta de sus doctorados.

\section{Agradecimientos}

Este trabajo fue posible gracias al financiamiento de la Universidad Nacional Autónoma de México (UNAM), a través del Programa de Becas Posdoctorales del Instituto de Investigaciones sobre la Universidad y la Educación (IISUE), realizado con la asesoría de los doctores Alejandro Canales Sánchez y Alejandro Márquez Jiménez.

Muchas gracias a todos los expertos que colaboraron con su tiempo y opiniones para realizar este trabajo, especialmente al Dr. Luis Ponce Ramírez, ex director de Posgrado de Conacyt y al equipo de esta dirección por el apoyo y colaboración brindada.

\section{Referencias}

Almanza, S. y Hernández, H. (2009). Vinculación ciencia-industria: sustento teórico metodológico para una nueva herramienta de planeación estratégica universitaria. En J. Basave y $\mathrm{M}$. Rivera (Coord.), Globalización, conocimiento y desarrollo. Teoría y estrategias de desarrollo en el contexto del cambio histórico mundial Tomo II (pp. 151-171). México: UNAM-Instituto de Investigaciones Económicas.

Arellano, D. (2012). Conclusiones. En S. Cárdenas, E. Cabrero, y D. Arellano (eds.). La difícil vinculación universidad-empresa en México ¿Hacia la construcción de la triple hélice? (pp. 251-256). México: Centro de Investigación y Docencia Económicas, A. C. 
Auriol, L. (2010). Careers of doctorate holders: Employment and mobility patterns. Recuperado de http://www.ub.edu/escola_doctorat/sites/ default/files/internacionalitzacio/OECD_ mobility_indicators.pdf

Bourner, T., Bowden, R. y Laing, S. (2001). Professional Doctorates in England. Studies in Higher Education, 26(1), 65-83. Doi: 10.1080/03075070124819.

Cabrero, E. (2012). Introducción. En S. Cárdenas, E. Cabrero, y D. Arellano (eds.). La dificil vinculación universidad-empresa en México ¿Hacia la construcción de la triple hélice? (pp. 9-21). México: Centro de Investigación y Docencia Económicas, A. C.

Chiteng, F. y Hendel, D. (2012). Emergence and Growth of Professional Doctorates in the United States, United Kingdom, Canada and Australia: A Comparative Analysis. Studies in Higher Education, 37(3), 345-364. Doi:10.10 80/03075079.2010.516356.

Consejo Mexicano de Estudios de Posgrado. (2017a). Posgrados. [Archivo de datos]. Recuperado de http://www.comepo.org.mx/

Consejo Mexicano de Estudios de Posgrado. (2017b). Segunda Declaratoria de Morelia sobre el posgrado nacional. Ceremonia de clausura del XXXI Congreso Nacional de Posgrado 2017, 22 de septiembre de 2017. Recuperado de http:// www.comepo.org.mx/images/xxxicnp/segundadeclaratoria-morelia-2017-comepo.pdf

Consejo Nacional de Ciencia y Tecnología. (2017). Padrón del Programa Nacional de Posgrados de Calidad. [Base de datos]. Recuperado de http:// svrtmp.main.conacyt.mx/ConsultasPNPC/ listar_padron.php

Consejo Nacional de Ciencia y Tecnología y Subsecretaría de Educación Superior de la Secretaría de Educación Pública. (2015). Marco de referencia para la evaluación y seguimiento de programas de posgrado presenciales, versión 6. Recuperado de http:// www.conacyt.gob.mx/index.php/becas-yposgrados/programa-nacional-de-posgrados- de-calidad/convocatorias-avisos-y-resultados/ convocatorias-cerradas-pnpc/9005-marcode-referencia-modalidad-escolarizada/file

Cuthbert, D. y Molla, T. (2015). PhD Crisis Discourse: A Critical Approach to the Framing of the Problem and some Australian "Solutions". Studies in Higher Education. 69, 33-53. Doi: 10.1007/s10734-014-9760-y.

De Sousa, B. (2004). La universidad en el siglo XXI, Argentina: Miño y Dávila.

Fernández, E. (2009). El sistema-mundo del capitalismo académico: procesos de consolidación de la universidad emprendedora. Educación, Policy Analysis Archives/Archivos Analiticos de Politicas Educativas, 17, 1-43. Recuperado de http://www. redalyc.org/articulo.oa?id=275019727020

Finkel, L. (2015). La omnipresencia de los weberianos en la sociología de las profesiones. En L. Finker, La sociología de las profesiones: legados y perspectivas, (Tesis doctoral). Recuperado de http://eprints.ucm.es/37718/

Gibbons, M. et al., (1997). Introducción. En M. Gibbons et al., (autores) La nueva producción del conocimiento. La dinámica de la ciencia y la investigación en las sociedades contemporáneas (pp. 11-29). España: Pomares-Corredor, S. A. Gómez, V. y Tenti, E. (1989). Universidady profesiones. Crisis y alternativas, Argentina: Miño y Dávila.

Kehm, B. y Teichler, U. (2016). Doctoral education and labor market: Policy, questions and data needs. En L. Gokhberg, N. Shmatko y L. Auriol (eds.). The science and technology labor force. The Value of Doctorate Holders and Development of Professional Careers (pp. 11-29). Francia: Springer International Publishing Switzerland.

Lester, S. (2007). Conceptualizing the Practitioner Doctorate. Studies in Higher Education, 29(6), 757-770. Recuperado de doi.org/10.1080/ 0307507042000287249

Loxley, A. y Seery, A. (2012). The Role of the Professional Doctorate in Ireland from the Student Perspective. Studies in Higher Education, 37(1), 3-17. Doi:10.1080/030750 79.2010 .489148 . 
Núñez-Valdez, K. y González, J. (2019). Hacia la estandarización de los perfiles de egreso de los programas de doctorado. Revista Digital de Investigación en Docencia Universitaria, 13(2), 3-18. Recuperado de doi.org/10.4067/S071850062019000500003

Núñez-Valdés, K., González, J. y Sarzoza, S. (2019). Expectativas de los Doctorandos Chilenos: Insumos para la Elaboración de un Perfil de Egreso Doctoral. Formación Universitaria, 12(5), 3-14. Recuperado de http://dx.doi. org/10.4067/S0718-50062019000500003

Pacheco, T. (1997). La investigación universitaria como profesión (valores sociales y científicos en su evaluación). En T. Pacheco y A. Díaz Barriga (Coord.). La profesión. Su condición social e institucional (pp. 151-172). México: UNAMCentro de Estudios sobre la Universidad.

Patiño, J. (2019). Análisis comparativo entre el doctorado profesional y de investigación en México. Revista Iberoamericana de Educación Superior, 10(28), 25-41. Recuperado de doi. org/10.22201/iisue.20072872e.2019.28.427

Patiño, J. (2017). Doctorados profesionales: concepciones y debate. Entrevista a Luis Ponce Ramírez. Revista Electrónica de Investigación Educativa, 19(4), 1-15. Recuperado de https:// redie.uabc. $\mathrm{mx} / \mathrm{redie} /$ article/view/1741

Patiño, J. (2016). Graduados de doctorado en México: factores asociados a su distribución por área de la ciencia y sector de sostenimiento. En J. Toro y M. Sánchez (Coord.). XXX Congreso Nacional de Posgrado. Expo Posgrado 2016. El posgrado como detonante de los procesos de innovación. (pp. 186-189). Recuperado de http://www.comepo.org.mx/images/xxxcnp/ memorias-xxx-congreso-nacional-posgradocomepo.pdf

Rodríguez, J. (2009). El nuevo capitalismo en la literatura económica y el debate actual. En A. Dabat y J. Rodríguez (Coord.). Globalización, conocimiento y desarrollo. La nueva economía global del conocimiento. Estructura y problemas
Tomo I (pp. 23-56). México: UNAM-Instituto de Investigaciones Económicas.

Sarfatti, M. (1988). Acerca de los expertos y la imposibilidad de haberlo dicho todo, Revista de Educación, 285, 151-189. Recuperado de http://www.mecd.gob.es/dctm/revistade\%20 educacion/articulosre1990/re 199007. pdf?documentId=0901e72b8136908c

Schildkraut, J. y Stafford, M. (2015). Researching Professionals or Professional Researchers? A Comparison of Professional Doctorate and PhD Programs in Criminology \& Criminal Justice. American Journal and Criminal Justice, 40(1), 183-198. Doi:10.1007/s12103-014-9240-z.

Stromquist, N. (2009). La profesión académica frente a las cambiantes expectativas sociales e institucionales". En N. Stromquist, (Coord.). La profesión académica en la globalización, México: ANUIES.

Taylor, J. (2004). The United Kingdom. En J. Sadlak (ed.). Doctoral Studies and Qualifications in Europe and the United States: Status and Prospects (pp. 231-258). Recuperado de http://unesdoc. unesco.org/images/0013/001364/136456e. pdf

Teichler, U. (2015). Educación y formación doctoral: una mirada por países y disciplinas. En M. De Ibarrola y L. Anderson (Coord.). La formación de nuevos investigadores educativos. Diálogos y debates (pp. 19-55). México: ANUIES.

Wildy, H., Peden, S. y Chan, K. (2015). The Rise of Professional Doctorates: Case Studies of the Doctorate in Education in China, Iceland and Australia. Studies in Higher Education, 40(5) 1-14. Doi:10.1080/03075079.2013.842968. 